\title{
The role of biochemistry and neurophysiology in the re- education of deglutition
}

\author{
Fellus Patrick* \\ Qualified specialist, Former Consultant for the Hospitals of Paris, France
}

\begin{abstract}
The transition from suction-type deglutition to dentition-type deglutition should spontaneously occur between ages 3 and 4 , but the genetic information is not always systematically expressed. The new program must then be engrammed by cortical or sub-cortical pathways.
\end{abstract}

\section{Introduction}

Suction-type deglutition, the physiological mode for swallowing saliva in the young infant, is a praxis developed in the brainstem in utero.

It will remain physiological as long as the primary dentition is not established and mastication has not begun.

But beginning at age 4, a new deglutition program is naturally implemented via a subcortical pathway in $60 \%$ of children: dentitiontype deglutition.

By the muscular force it generates, it promotes optimal growth of the maxillae [1].

But endogenous or innate factors can only provide the potential. "They will only be expressed if adequate exogenous conditions occur in a timely manner" (JP Changeux) [2,3]. Dawson Church states that "genes are activated or inactivated by our beliefs, emotions and attitudes."

There are two principal causes for the persistence of suction-type deglutition:

- the child has never had the opportunity to discover this new function

- the child has discovered it, but the limbic system, the gateway to engramming a new program, has not retained it, usually for psychological reasons (immaturity, thumb, pacifier, bottle, food too soft).

Most children requiring orthodontic treatment are found in the latter category.

The re-education of orofacial functions is consequently a necessity accepted by nearly all practitioners during orthodontic treatment, but it is necessary to properly understand both neuroanatomy and physiology to achieve reproducible and controllable results.

\section{Deglutition re-education \\ What strategy to implement? \\ - hope that the normalization of dental arches will lead to a change in function.}

This is more feasible when wearing a removable retainer. The introduction of an element modifying proprioceptive sensations will automatically lead to a change in the afferent message, and consequently the efferent message [4]. The anatomical part having been modified by treatment, the correct program can install itself naturally, but without control, the new praxis may also remain dysfunctional. However, care must be taken with bonded retainers.

If the functional modification occurs, there are no negative consequences, but if the dysfunction remains, the pathological muscular force will be iatrogenic, no longer in the dental alignment but in the supporting bone tissue, which may be the source of subsequent periodontal disease.

- use of functional devices, usually at night, lingual envelope-type (NLE) devices intended to modify the lingual posture, Robin device, lingual elevator, Farrell or Bergensen-type gutters. However, the results remain haphazard, as long as the dysfunctional commands are not inhibited, aside from the fact that wearing these devices may be difficult for the child to deal with and rejected by the limbic system.

- consult professionals, such as speech therapists or physiotherapists.

The child must initially become aware of what he/she is doing, then the maneuver he/she should be doing, and its repetition should enable automation.

Eric Kandel, Nobel Prize in Medicine recipient in 2000 for his work on the transition from short-term to long-term memory, demonstrated in these cases an increase in the activity of neurotransmitters in the synapses involved, but we remain in the domain of short-term memory $[5,6]$.

"Memory is not based on the properties of nerve cells as such, but on the nature of the connections between neurons and how they process the sensory information received": learning consists of tracing new circuits, and this plasticity occurs either by reconfiguring existing programs or creating new ones (Figure 1 and Table 1).

Correspondence to: Fellus Patrick, Qualified specialist, Former Consultant for the Hospitals of Paris, France, E-mail: fellusp@wanadoo.fr

Key words: deglutition, short-term memory, procedural memory

Received: June 09, 2017; Accepted: June 21, 2017; Published: June 24, 2017 
Table 1. Reconfiguring existing circuits.

\begin{tabular}{|c|c|}
\hline Neurone 1 & Neuron 1 \\
\hline Message nerveux & Nerve message \\
\hline Neurone 2 & Neuron 2 \\
\hline Nouvelles connexions & New connections \\
\hline Forte transmission du message nerveux & Strong transmission of nerve message \\
\hline Faible transmission du message nerveux & Weak transmission of nerve message \\
\hline Connexion entre les neurones 1 and 2 & Connection between neurons 1 and 2 \\
\hline
\end{tabular}

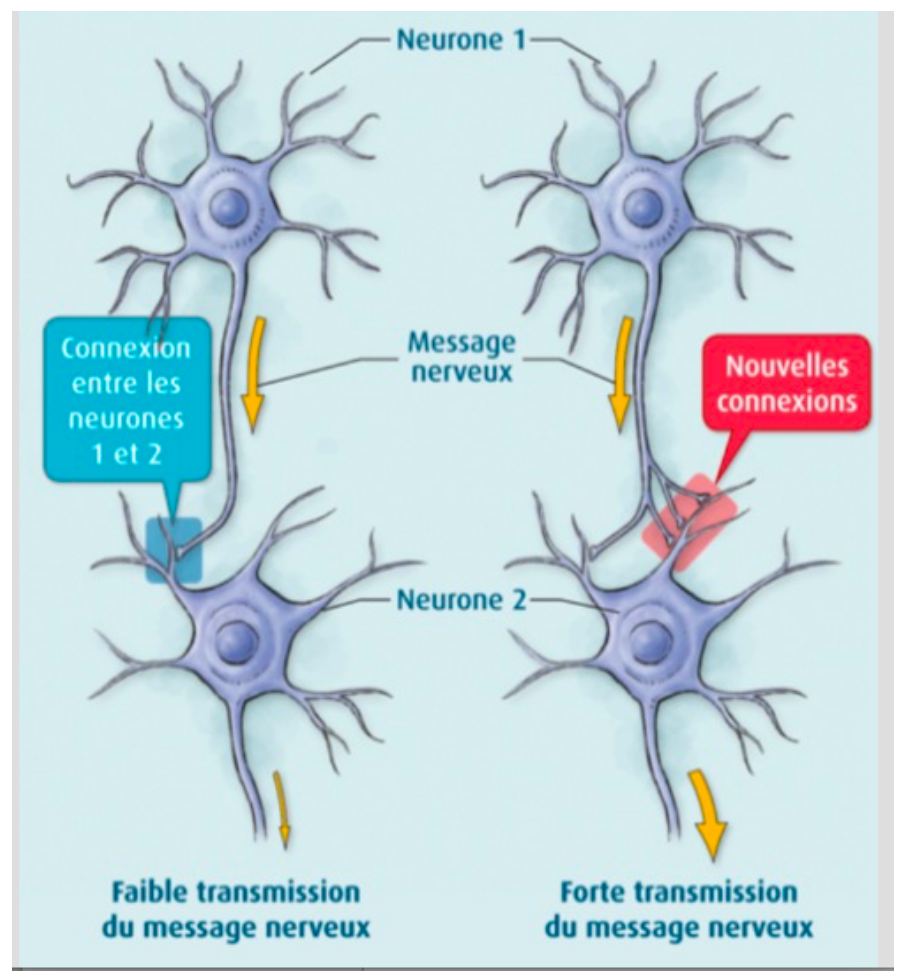

Figure 1. Eric Kandel Diagram.

Understanding the transition from short-term memory to longterm memory was clarified by the work of Kandell in Aplysia, by reconstituting a simplified neural circuit: a single sensory neuron connected to a single motor neuron:

- A slight stimulus releases neurotransmitters at the synapse, but the nucleus is not involved; this is short-term memory (weekly speech therapy sessions). This information will remain available only briefly.

- If the stimuli are repeated soon after, a dialogue occurs between the synapse and the nucleus to activate $\mathrm{CREB}^{\star}$ and produce a new protein, essential for the transition to long-term memory. This new $\mathrm{CPEB}^{* *}$ protein present in the synapse will function as a prion and permanently ensure the transmission of the message.

${ }^{*}$ CREB (Cyclic AMP Response Element-Binding Protein), a protein that activates the genes responsible for long-term memory. CREB 1 is the activator and CREB is the 2 inhibitor.

${ }^{* *}$ CPEB: (Cytoplasmic Polyadenylation Element-Binding Protein), a transcriptional regulatory protein in the synapse which contributes to the stabilization of long-term memory.

\section{Creating new circuits}

A highly emotional state can short-circuit normal constraints and produce a sufficient quantity of MAP-kinase ${ }^{\star}$ molecules, which will be sent to the nucleus to inactivate CREB-2** molecules and facilitate CREB- $1^{\star *}$ activation and direct imprinting of this experience in longterm memory

${ }^{*}$ MAP-kinase kinase: acts in conjunction with protein kinase A, to initiate long-term memorization.

- FroggyMouth is a device worn a rather short time, 15 minutes a day while watching television (a reward recognized by the limbic system), which will force the child to discover a new deglutition method via the sub-cortical pathway; thus, not by stimulating neurotransmitter activity, but by creating new synapses.

In fact, by no longer being able to close the lips, the child will be unable to swallow by suction, aspirating between the anterior and posterior mouth, triggering an abrupt and immediate reaction in the brainstem: find a new deglutition program.

The simultaneous contraction of the levator muscles of the mandible in a stable and comfortable dental occlusion with those of the soft palate and styloglossus will allow a peristaltic movement of the tongue (provided that the anatomical environment is compatible) and disconnect the tongue-lips synkinesis.

This new deglutition program will be immediately integrated into long-term memory by the creation of a new neural circuit.

But this is only the first step, which is necessary but not sufficient for the transition to automation.

\section{Automation}

The child will then have two programs for swallowing saliva, and just as on a computer when there are two programs, it is the activation of one or another icon that initiates its execution (Figures 2 and 3).

The therapist should therefore monitor the resting posture for relaxation of the perioral muscles and dental occlusion upon swallowing.

Control by the trigeminal nerve, solicited at this step, will replace control by the facial nerve and inhibit the role of the latter.

This necessary inhibition of the faulty circuit is fundamental to automation of the correct program. Only FroggyMouth allows this double action. The trigeminal nerve, which also controls the respiration centers in the pontine tegmentum through its sensory nucleus, will promote restoration of nasal respiration, allowing the tongue to adopt a high posture posteriorly (the lingual dome).

"This dual posterior and occlusal functional necessity of the tongue, too often forgotten by re-educators of orofacial function, is likely one of the causes of the too-frequent failures of re-education" (Delaire) [7].

Similarly, contraction of the tensor tympani muscle, innervated by the trigeminal nerve, will allow ventilation of the middle ear by dilating the Eustachian tube, decreasing serous otitis problems Control may be transferred to the parents, who need to observe lip posture 5 times/ day, and who then congratulate or correct the child (Figures 4 to 8). These two actions are not similar, involving cortico-cortical circuits that traverse the basal ganglia and cortico-cortical circuits that traverse the cerebellum [8].

Wearing FroggyMouth starting at age 3 has no contraindications.

\section{Conclusion}

Early normalization of orofacial functions, regardless of the technique chosen, will enable a three-step preventive approach: 


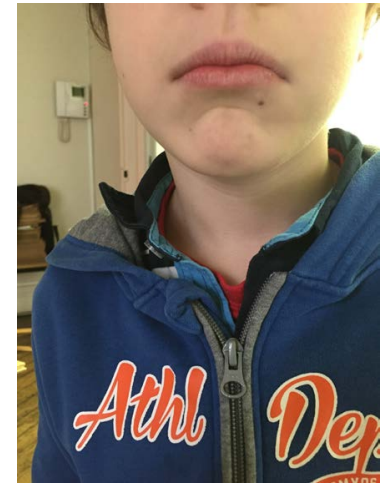

Figure 2. The suction-type deglutition icon is activated by the facial nerve: "My lips are contracted, my teeth do not touch."

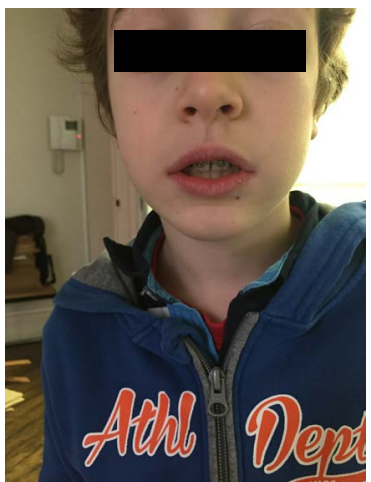

Figure 3. The dentition-type deglutition icon: "my lips are relaxed, my molars are in occlusion" is activated by the trigeminal nerve, whichallows not onlymolar occlusion but also the protection of the tongue from biting, due to the abundance of trigeminal nerve endings in its epitheliallining.
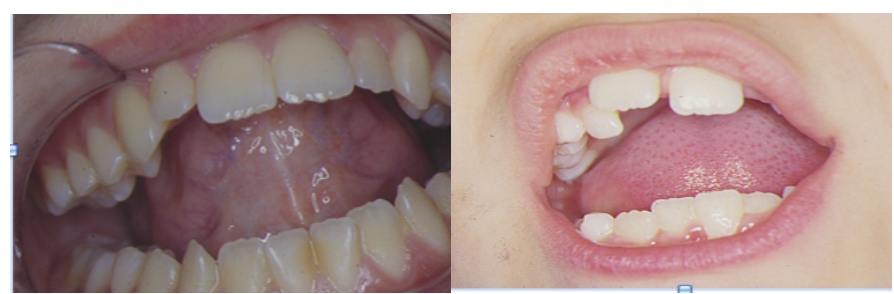

Figure 4. Contrary to what speech therapists suggest, attention needs to be paid not to the tip of the tongue, but rather to its posterior portion. Obsessedwith the sensorysearch for the retro-incisive papilla, the child risks raising the tip of the tongue, leading to lowering of the posterior portion, which will prevent the involvement of the styloglossus, the levator muscle of the lingual dome.

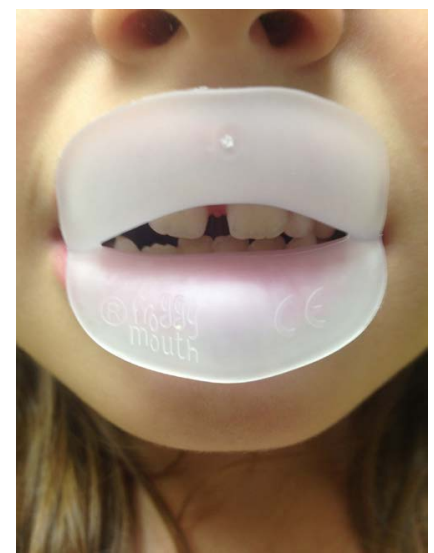

Figure 5. Worn for 15 min daily while watching television, FroggyMouth allows relaxation of all anterior facial muscles.

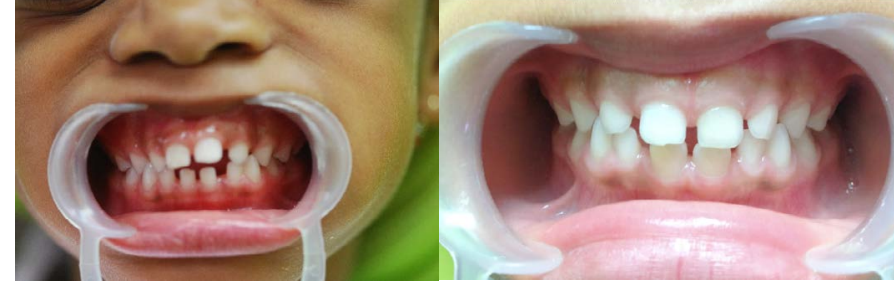

Figure 6. Spontaneous improvement of an incisor gap after wearing Froggy Mouth for one month.

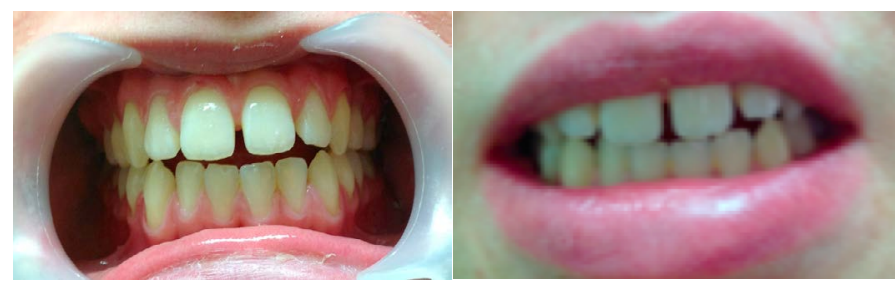

Figure 7. Resumption of treatment with only Froggy Mouth after repetition of a previous orthodontic treatment.

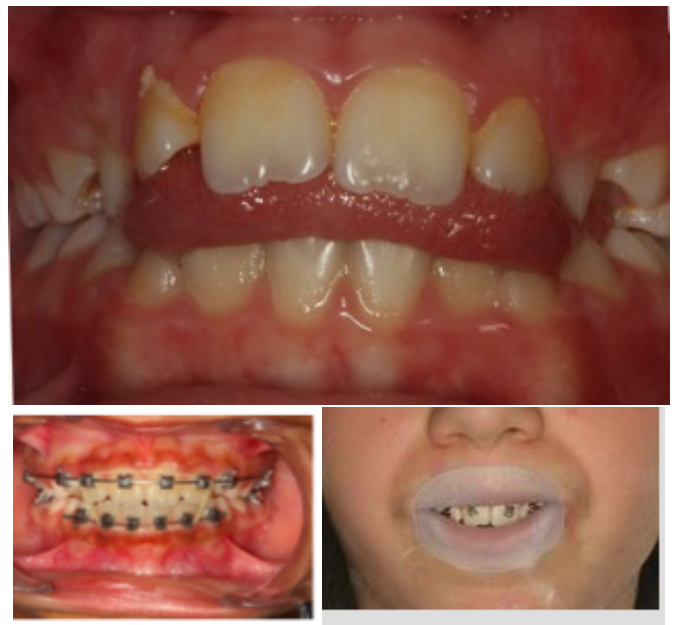

Figure 8. Case treated by Gérard Altounian.

-prevent deformities from appearing,

-if they occur, prevent them from worsening,

\section{References}

1. Patrick F (2003) Orthodontie précoce en denture temporaire Cdp

2. Jean-Pierre C (1983) L'homme neuronal Fayard

3. Gérard C (2010) Les oralités humaines Doin.

4. Gérard C (2015) Oralité du fœtus Sauramps Médical

5. Arthur G (1996) Neurosciences. Piccin.

6. Eric K (2011) A la recherche de la mémoire. Odile Jacob.

7. Patrick F, Waddah S, Lalauze-Pol R (2016) De la dysfonction à la dysmorphose. Apport de Froggy mouth. Edition Orthopolis.

8. Fournier M, Girard M (2013) Acquisition and sustainment of automatic reflexes in maxillofacial rehabilitation. Orthod Fr 84: 287-294. [Crossref]

Copyright: (C2017 Patrick F. This is an open-access article distributed under the terms of the Creative Commons Attribution License, which permits unrestricted use, distribution, and reproduction in any medium, provided the original author and source are credited. 